\title{
MATHEMATICAL AND NUMERICAL STUDY OF A DUSTY KNUDSEN GAS MIXTURE
}

\author{
FRÉDÉRIQUE CHARLES AND FRANCESCO SALVARANI
}

\begin{abstract}
We consider a mixture composed of a gas and dust particles in a very rarefied setting. Whereas the dust particles are individually described, the surrounding gas is treated as a Knudsen gas, in such a way that interactions occur only between gas particles and dust by means of diffuse reflection phenomena. After introducing the model, we prove existence and uniqueness of the solution and provide a numerical strategy for the study of the equations. At the numerical level, we focus our attention on the phenomenon of energy transfer between the gas and the moving dust particles.
\end{abstract}

\section{INTRODUCTION}

The study of particle systems under the influence of a surrounding fluid is a very active research field, with many possible applications. Among them, we can quote the study of the dynamics of gases inside a microelectromechanical system (MEMS).

MEMS are very small devices (for example, accelerometers, gyroscopes, pressure sensors and micro ultrasound transducers) having many commercial applications. Usually, they are made up of components between 1 to $100 \mu \mathrm{m}$ in size and use air as lubricant between their moving parts. It is apparent that, in this setting, the usual approximations of macroscopic fluid dynamics are no longer verified, especially when the gas pressure is under a given threshold, which depends on the precise geometry of the problem. In such a situation, indeed, the mean free path of the gas molecules can be larger than the macroscopic size of the device and the kinetic approach is necessary for a correct description of the physical system.

Kinetic models describe the collective behavior of a large number of elementary particles in terms of their distribution number density in phase space. The effects of binary interactions between particles are treated at the microscopic level as simple binary collision (or scattering) events, whose time scale is much shorter than the time scale of the evolution of the distribution number density. At the microscopic level, the description only involves a few fundamental laws (such as the conservation of momentum and the conservation of kinetic energy - when the collisions between particles are of elastic type). For this reason, kinetic models depend much less on phenomenological laws than most models of continuum mechanics. In particular, the main macroscopic collective features of the system can be rigorously deduced by means of an appropriate limiting procedure on an ab initio kinetic model, rather than heuristically introduced in the macroscopic model.

The working hypotheses of the article are the following. We consider a system composed of a very rarefied gas of small light molecules - such as air - interacting with heavy and big (with respect to the order of magnitude of small molecules) bodies (for example, dust particles). The mixture evolves in a domain with a reference length of about $10 \mu \mathrm{m}$ (which is a typical length scale of MEMS), in a medium vacuum (i.e. with a pressure of about $10 \mathrm{~Pa}$ or less), observed on a short-range time scale.

In this setting, at a temperature of $273 \mathrm{~K}$, the mean free path of a gas such as $\mathrm{O}_{2}$ or $\mathrm{N}_{2}$ is of about $500 \mu \mathrm{m}$, and under such hypotheses the gas can be treated with a good approximation as a non-collisional gas. Since we are interested in lubrication effects, we do not introduce different density functions for each species of air, because the physical properties of the constituents are very similar for our purposes.

Date: December 14, 2018.

Key words and phrases. Knudsen gas, moving domain, particle method. 
Whereas the gas is described by a density function, we suppose that the number of dust particles is very small, in such a way that it is possible to follow them individually. We suppose moreover that the motion and the other physical properties (such as the temperature) of dust particles are not influenced by the action of the gas molecules. This simplification is reasonable if we work on a small time scale and when the ratio between the mass of the gas molecules and the mass of the dust particles is very small.

Moreover, we retain only the interactions between dust and gas particles. This simplification is reasonable for very rarefied gases and strongly reduces the cost of the numerical simulation.

At the mathematical level, our model describes the system as a Knudsen (or non-collisional) gas in a moving domain. The studies in this setting are increasing in the last years. Among the contributions based on kinetic theory techniques, we quote [5] on the diffusion approximation of a non-interacting gas and $[1,13]$ which deal with the approach to equilibrium for Knudsen gases.

The analysis of the long-time behavior in the situation of a locally non-interacting (degenerate) system has been carried out in $[2,3,4,9,10]$, and the study of a Knudsen system in a moving domain has been the subject of [8] (which has subsequently been generalized in [11]).

This article is organized as follows. In the next section, we outline our model and precise the hypotheses and the approximations of our study. In Section 3 we provide a result about the existence and uniqueness of the solution of the model and in Section 4 we describe the numerical method used in the simulations. The numerical results are finally detailed in Section 5 .

\section{Formalization of the PRoblem AND DESCRIPTION OF THE MODEL}

We model our problem by considering a mixture composed of a rarefied gas and a finite number of heavy dust particles in a perfectly reflecting container $D \subset \mathbb{R}^{\ell}, \ell=2,3$. We suppose that the dust particles - whose number is arbitrary - are perfectly spherical, with radius $r>0$. We moreover assume that the size of the dust particles is larger than the mean free path of the rarefied gas, but small with respect to the size of the container. We hence neglect the interactions between gas molecules and take into account only collisions between the rarefied gas and the dust particles (we hence work in the framework of a Knudsen gas). Since the gas is very rarefied and the mass of the gaseous molecules is very small compared with the mass of the dust particles, we suppose that the small gas molecules are influenced by the heavy dust particles, but that the converse is not true. Hence, the behavior of the dust particles can be described by means of the Newton laws of classical mechanics, as the interaction between spheres.

Suppose that there exist $N_{d} \in \mathbb{N}$ dust particles and that their motion law is completely known. We can hence describe their space-time evolution through the given motion law of their centers $\xi_{i}=\xi_{i}(t)$ and the corresponding $\ell \times \ell$ rotation matrices $A_{i}=A_{i}(t), i \in \mathbb{N}$. Obviously, the motion laws of the particles take into account the fact that the particles do not overlap and do not leave the container $D$ through appropriate constraints.

As it is well known, the rotation matrices are real and orthonormal. In what follows, we suppose moreover that the motion laws of the centers of the spheres and the rotation matrices are regular with respect to $t$ (at least of class $C^{1}$ ).

For any $i=1, \ldots, N_{d}$, denote with $B_{r}\left(\xi_{i}(t)\right)$ the region occupied by the dust particles labelled by the index $i$, i.e.

$$
B_{r}\left(\xi_{i}(t)\right)=\left\{x \in \mathbb{R}^{\ell}:\left\|x-\xi_{i}(t)\right\|<r\right\} .
$$

Hence, we introduce the time $T_{1}$ which guarantee the non-overlapping of dust particles

$$
T_{1}=\sup \left\{t \geq 0: \forall s \in\left[0, t\left[, B_{r}\left(\xi_{j}(s)\right) \cap B_{r}\left(\xi_{i}(s)\right)=\emptyset \text { for all } j, i=1, \ldots, N_{d}, j \neq i\right\}\right.\right.
$$

and the time $T_{2}$ which guarantee the non-exit of dust particles out of the domain

$$
T_{2}=\sup \left\{t \geq 0: \forall s \in\left[0, t\left[\inf _{x \in \partial D}\left\|x-\xi_{i}(s)\right\| \geq r \quad \text { for all } i=1, \ldots, N_{d}\right\} .\right.\right.
$$


For the sake of simplicity, we suppose that $t \in\left[0, T\left[\right.\right.$, where $\left.\left.T \leq \min \left(T_{1}, T_{2}\right) \in\right] 0,+\infty\right]$. For $t \in\left[0, T\left[\right.\right.$, we then define the time-dependent region $\Omega^{t}$ occupied by the Knudsen gas at time $t$ as

$$
\Omega^{t}=D \backslash \sum_{i=1}^{N_{d}} B_{r}\left(\xi_{i}(t)\right),
$$

and the collective reflecting boundary of the dust particles $\Gamma^{t}$ as

$$
\Gamma^{t}=\sum_{i=1}^{N_{d}} \partial B_{r}\left(\xi_{i}(t)\right) .
$$

Hence, the boundary of $\Omega^{t}$ can be described as the sum of two parts: the boundary $\Gamma^{t}$ and the boundary of the container $\partial D$, i.e.

$$
\partial \Omega^{t}=\Gamma^{t} \cup \partial D
$$

Note that, as we will see later on, the boundary conditions on $\Gamma^{t}$ and on $\partial D$ are different.

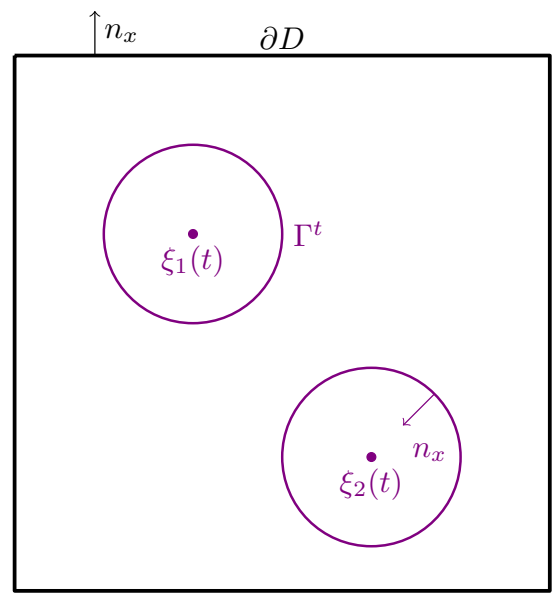

FiguRE 1. Graphical description of the problem.

The motion of the dust particles being known, it is possible to deduce the velocity law of each point of the boundary at a given time $t$. For simplifying the notation, we define a field $c: \mathbb{R}^{+} \times \mathbb{R}^{\ell} \rightarrow \mathbb{R}^{\ell}$ which gives the local velocity of each point $x \in \partial \Omega^{t}$ for any $t \in[0, T[$ (obviously, for any $x \in \partial D$, we have that $c(t, x)=0$ ).

Let $(x, v) \in \Omega^{t} \times \mathbb{R}^{\ell}$. In order to describe the time evolution of the Knudsen gas which moves in the allowed portion of the domain $\Omega$ (represented by the time-dependent family of domains $\Omega^{t}$ for all $t \geq 0$ ), we introduce a distribution function $f:=f(t, x, v)$, whose physical meaning is the following: if $X \subset \Omega^{t}$ and $V \subset \mathbb{R}^{\ell}$, the integral

$$
\int_{X \times V} f(t, x, v) \mathrm{d} x \mathrm{~d} v
$$

represents the number of gas molecules that, at time $t$, have position $x \in X$ and velocity $v \in V$. For simplicity, the total mass of the gas will be normalized.

We suppose moreover that the initial conditions have support contained in $\Omega^{0}$, and that the only mechanisms of deviation of the gas particles from the free flow are given by the interaction with the boundary $\partial \Omega^{t}$. More precisely, we will suppose perfectly specular reflexion for the particles hitting $\partial D$ and diffuse reflexion conditions for the interaction between gaseous particles and dust. Throughout this article, we suppose that the outward normal $n_{x} \in \mathbb{S}^{\ell-1}$ 
of the domain $\partial \Omega^{t}$, originated in $x \in \partial \Omega^{t}$, is directed outside the region occupied by the gas (in particular, $n_{x}$ is directed inside the dust particles).

Denote, for all $t \in\left[0, T\left[\right.\right.$ and for all $x \in \partial \Omega^{t}$,

$$
\mathbb{V}_{t, x}^{-}:=\left\{v \in \mathbb{R}^{\ell}:(v-c(t, x)) \cdot n_{x}<0\right\}
$$

and consider the following kernels specifying the conditions on the boundary:

- the specular reflexion kernel

$$
k(t, x, v, w)=\delta\left(w-v+2\left(v \cdot n_{x}\right) n_{x}\right), \quad(t, x, v, w) \in \mathbb{R}^{+} \times \partial D \times \mathbb{R}^{\ell} \times \mathbb{R}^{\ell} ;
$$

- the diffuse reflexion kernel

$$
k(t, x, v, w)=M_{T_{p}}(v-c(t, x))\left|(w-c(t, x)) \cdot n_{x}\right|, \quad(t, x, v, w) \in \mathbb{R}^{+} \times \Gamma^{t} \times \mathbb{R}^{\ell} \times \mathbb{R}^{\ell},
$$

where $M_{T_{p}}$ is the centered Maxwellian function with zero mean velocity and fixed temperature $T_{p}$ :

$$
M_{T_{p}}(w)=\frac{1}{\left(2 \pi T_{p}\right)^{\ell / 2}} e^{-|w|^{2} / 2 T_{p}}, \quad w \in \mathbb{R}^{\ell} .
$$

Note that $M_{T_{p}}$ satisfies the flux normalization conditions

$$
\int_{\mathbb{R}^{\ell} \backslash \mathbb{V}_{t, x}^{-}} M_{T_{p}}(v-c(t, x))(v-c(t, x)) \cdot n_{x} \mathrm{~d} v=1 \quad \text { for all } x \in \Gamma^{t} .
$$

The time evolution of $f$ is hence governed by the following partial differential equation:

$$
\frac{\partial f}{\partial t}+v \cdot \nabla_{x} f=0 \quad(t, x, v) \in \mathbb{R}^{+} \times \Omega^{t} \times \mathbb{R}^{\ell}
$$

with normalized non-negative initial data

$$
f(0, x, v)= \begin{cases}f^{\text {in }}(x, v) & \text { if }(x, v) \in \Omega^{0} \times \mathbb{R}^{\ell} \\ 0 & \text { otherwise }\end{cases}
$$

where $f^{\text {in }} \in L^{\infty}\left(\Omega^{0} \times \mathbb{R}^{\ell}\right),\left\|f^{\text {in }}\right\|_{L^{1}\left(\Omega^{0} \times \mathbb{R}^{\ell}\right)}=1$ and boundary conditions:

$$
f(t, x, v)=\int_{\mathbb{R}^{\ell} \backslash \mathbb{V}_{t, x}^{-}} k(t, x, v, w) f(t, x, w) \mathrm{d} w, \quad(t, x, v) \in(0, T) \times \partial \Omega^{t} \times \mathbb{V}_{t, x}^{-} .
$$

An useful tool for studying the initial-boundary value problem (2.5)-(2.6)-(2.7) is the concept of backward interaction time, whose definition is the following.

Definition 2.1. The backward interaction time $\tau_{\Omega^{t}}(x, v)$ for a particle starting from $x \in \Omega^{t}$ in the direction $v \in V$, is defined as

$$
\tau_{\Omega^{t}}(x, v)=\inf \left\{\theta>0: x-\theta v \in \Gamma^{t-\theta} \cup \partial D\right\} .
$$

If the set $\Theta:=\left\{\theta>0: x-\theta v \in \Gamma^{t-\theta} \cup \partial D\right\}$ is empty, then $\tau_{\Omega^{t}}(x, v)=+\infty$.

\section{Existence AND UniQUeness}

Our first step is the study of the existence and uniqueness properties for the initial-boundary value problem (2.5)-(2.6)-(2.7).

We begin with an a priori result:

Lemma 3.1. Let $\eta>0$ and $f^{\text {in }} \in L^{2}\left(\Omega^{0} ; L^{2}\left(\mathbb{R}^{\ell}, e^{\eta|v|^{2}} \mathrm{~d} v\right)\right)$ a non-negative initial condition. If there exists a solution $f=f(t, x, v)$ of the initial-boundary value problem (2.5)-(2.6)-(2.7), then

$$
\|f(t, \cdot, \cdot)\|_{L^{2}\left(\Omega^{t} ; L^{2}\left(\mathbb{R}^{\ell}, e^{\eta|v|^{2}} \mathrm{~d} v\right)\right)} \leq\left\|f^{\mathrm{in}}\right\|_{L^{2}\left(\Omega^{0} ; L^{2}\left(\mathbb{R}^{\ell}, e^{\eta|v|^{2}} \mathrm{~d} v\right)\right)},
$$

for a.e. $t \in(0,+\infty)$. 
Proof. By Reynolds theorem, we have

$$
\frac{d}{d t} \int_{\Omega^{t} \times \mathbb{R}^{\ell}} e^{\eta|v|^{2}} f^{2} \mathrm{~d} x \mathrm{~d} v=\int_{\Omega^{t} \times \mathbb{R}^{\ell}} e^{\eta|v|^{2}}\left(f^{2}\right)_{t} \mathrm{~d} x \mathrm{~d} v+\int_{\partial \Omega^{t} \times \mathbb{R}^{\ell}} e^{\eta|v|^{2}} f^{2}\left(c \cdot n_{x}\right) \mathrm{d} S(x) \mathrm{d} v .
$$

Multiply Equation $(2.5)$ by $e^{\eta|v|^{2}} f$, and then integrate with respect to the variables $(x, v)$ in $\Omega^{t} \times \mathbb{R}^{\ell}$. We obtain that

$$
\begin{aligned}
\int_{\Omega^{t} \times \mathbb{R}^{\ell}} e^{\eta|v|^{2}}\left(f^{2}\right)_{t} \mathrm{~d} x \mathrm{~d} v & =-\int_{\Omega^{t} \times \mathbb{R}^{\ell}} e^{\eta|v|^{2}} \nabla_{x} \cdot\left(v f^{2}\right) \mathrm{d} x \mathrm{~d} v \\
& =-\int_{\partial \Omega^{t} \times \mathbb{R}^{\ell}} e^{\eta|v|^{2}} f^{2}\left(v \cdot n_{x}\right) \mathrm{d} S(x) \mathrm{d} v,
\end{aligned}
$$

where $\mathrm{d} S(x)$ denotes the surface element on $\partial \Omega^{t}$ centered at $x \in \partial \Omega^{t}$. Hence we get

$$
\frac{d}{d t} \int_{\Omega^{t} \times \mathbb{R}^{\ell}} e^{\eta|v|^{2}} f^{2} \mathrm{~d} x \mathrm{~d} v=-\int_{\mathbb{R}^{\ell}} e^{\eta|v|^{2}} f^{2}(t, x, v)(v-c) \cdot n_{x} \mathrm{~d} v \leq 0
$$

where the last inequality is a consequence of Equation (A.267) in [12] with $F(s)=s^{2}$, which gives a generalization of Darrozes-Guiraud's Lemma [7] for domains with moving walls (note that we are using the opposite convention on the direction of $n_{x}$, see [12], Section 1.6.1).

An immediate consequence of the previous a priori estimate is the following result about the uniqueness of a solution for the initial-boundary value problem (2.5)-(2.6)-(2.7).

Corollary 3.2. Let $\eta>0$ and $f^{\text {in }} \in L^{2}\left(\Omega^{0} ; L^{2}\left(\mathbb{R}^{\ell}, e^{\eta|v|^{2}} \mathrm{~d} v\right)\right)$ a non-negative initial condition. If there exists a solution $f=f(t, x, v)$ of the initial-boundary value problem (2.5)-(2.6)-(2.7), then it is unique.

Proof. Suppose that there exist two different solutions $\hat{f}$ and $\tilde{f}$ of the initial-boundary value problem (2.5)-(2.6)-(2.7). Hence, by linearity, the difference $h=\hat{f}-\tilde{f}$ is a solution of (2.5)(2.6)-(2.7) with initial condition $h(0, x, v)=0$ for a.e. $(x, v) \in \Omega^{0} \times \mathbb{R}^{\ell}$.

By (3.1), it means that

$$
\|h(t, \cdot, \cdot)\|_{L^{2}\left(\Omega^{t} ; L^{2}\left(\mathbb{R}^{\ell}, e^{\eta|v|^{2}} \mathrm{~d} v\right)\right)} \leq 0,
$$

i.e. that $\hat{f}=\tilde{f}$ for a.e. $(x, v) \in \Omega^{t} \times \mathbb{R}^{\ell}, t \in(0, T)$.

The uniqueness of the solution being already known by Corollary 3.2, we investigate now the existence of a solution, which will be built by using an approximating sequence. In conclusion, the following theorem holds.

Theorem 3.3. Let $f^{\text {in }} \in L^{\infty}\left(\Omega^{0} \times \mathbb{R}^{\ell}\right) \cap L^{2}\left(\Omega^{0} ; L^{2}\left(\mathbb{R}^{\ell}, e^{\eta|v|^{2}} \mathrm{~d} v\right)\right)$, $f^{\text {in }} \geq 0$ for a.e. $(x, v) \in$ $\Omega^{0} \times \mathbb{R}^{\ell}$ and $\left.\left.T \in\right] 0, \min \left(T_{1}, T 2\right)\right]$, where $T_{1}$ and $T_{2}$ are defined by (2.1) and (2.2). Then there exists one and only one non-negative weak solution $f \in L^{\infty}\left((0, T) ; L^{2}\left(\Omega^{t} ; L^{2}\left(\mathbb{R}^{\ell}, e^{\eta|v|^{2}} \mathrm{~d} v\right)\right)\right.$ of the initial-boundary value problem (2.5)-(2.6)-(2.7).

Proof. Consider the auxiliary problem for the function $g: \mathbb{R}^{+} \times \Omega^{t} \times \mathbb{R}^{\ell} \rightarrow \mathbb{R}$

$$
\frac{\partial g}{\partial t}+v \cdot \nabla_{x} g=0, \quad(t, x, v) \in \mathbb{R}^{+} \times \Omega^{t} \times \mathbb{R}^{\ell},
$$

with initial data

$$
g(0, x, v)= \begin{cases}f^{\text {in }}(x, v) & \text { if }(x, v) \in \Omega^{0} \times \mathbb{R}^{\ell} \\ 0 & \text { otherwise }\end{cases}
$$

where $f^{\text {in }} \in L^{\infty}\left(\Omega^{0} \times \mathbb{R}^{\ell}\right)$, and boundary conditions

$$
g(t, x, v)=\Phi(t, x, v)
$$

for a.e. $(x, v) \in \partial \Omega \times \mathbb{V}_{t, x}^{-}$, where $\Phi$ is a given function such that

$$
\Phi \in L^{\infty}\left((0, T) \times\left(\partial \Omega^{t} \times \mathbb{V}_{t, x}^{-}\right)\right) .
$$


By applying the method of characteristics, it is easy to show that problem (3.2)-(3.3)-(3.4) has a unique weak solution, given by

$$
g(t, x, v)=f^{\mathrm{in}}(x-v t, v) \mathbb{1}_{\left\{\tau_{\Omega^{t}}(x, v)>t\right\}}+\Phi\left(t, x^{*}, v\right) \mathbb{1}_{\left\{\tau_{\Omega^{t}}(x, v)<t\right\}},
$$

where $x^{*}=x-\tau_{\Omega^{t}}(x, v) v$.

The explicit formula of the solution $g$ allows to deduce that

$$
\|g\|_{L^{\infty}\left((0, T) \times \Omega^{t} \times \mathbb{R}^{\ell}\right)} \leq \max \left\{\left\|f^{\mathrm{in}}\right\|_{L^{\infty}\left(\Omega^{0} \times \mathbb{R}^{\ell}\right)},\|\Phi\|_{L^{\infty}\left((0, T) \times\left(\partial \Omega^{t} \times \mathbb{V}_{t, x}^{-}\right)\right)}\right\} .
$$

We now consider the sequence $\left\{f_{n}\right\}_{n \in \mathbb{N}}$ of functions, such that

$$
f_{1}(t, x, v)=0 \text { for a.e. }(t, x, v) \in[0, T) \times \bar{\Omega}^{t} \times \mathbb{R}^{\ell}
$$

and, for all $n \in \mathbb{N}, n \geq 2, f_{n}$ are the solutions of the following initial-boundary value problems:

$$
\frac{\partial f_{n}}{\partial t}+v \cdot \nabla_{x} f_{n}=0, \quad(t, x, v) \in \mathbb{R}^{+} \times \Omega^{t} \times \mathbb{R}^{\ell},
$$

with initial data

$$
f_{n}(0, x, v)= \begin{cases}f^{\text {in }}(x, v) & \text { if }(x, v) \in \Omega^{0} \times \mathbb{R}^{\ell} \\ 0 & \text { otherwise }\end{cases}
$$

and boundary conditions

$$
f_{n}(t, x, v)=\int_{\mathbb{R}^{\ell} \backslash \mathbb{V}_{t, x}^{-}} k(t, x, v, w) f_{n-1}(t, x, w) \mathrm{d} w, \quad(t, x, v) \in(0, T) \times \partial \Omega^{t} \times \mathbb{V}_{t, x}^{-} .
$$

These equations have exactly the same form of (3.2)-(3.3)-(3.4). Since $f_{1}(t, x, v)=0$ on $\partial \Omega^{t} \times \mathbb{R}^{\ell}$, we immediately have that

$$
f_{2}(t, x, v)=f^{\text {in }}(x-v t, v) \mathbb{1}_{\left\{\tau_{\Omega^{t}}(x, v)>t\right\}}
$$

for a.e. $(t, x, v) \in(0, T) \times \Omega^{t} \times \mathbb{R}^{\ell}$ and

$$
\begin{aligned}
f_{n}(t, x, v)= & \mathbb{1}_{\left\{\tau_{\Omega^{t}}(x, v)>t\right\}} f^{\mathrm{in}}(x-v t, v) \\
& +\mathbb{1}_{\left\{\tau_{\Omega^{t}}(x, v)<t\right\}} \int_{\mathbb{R}^{\ell} \backslash \mathbb{V}_{t, x^{*}}^{-}} k\left(t, x^{*}, v, w\right) f_{n-1}\left(t, x^{*}, w\right) \mathrm{d} w \quad n \geq 2
\end{aligned}
$$

for a.e. $(t, x, v) \in(0, T) \times \Omega^{t} \times \mathbb{R}^{\ell}$.

It is easy to see by iteration and by (2.4) that

$$
0 \leq f_{n} \leq\left\|f^{\text {in }}\right\|_{L^{\infty}\left(\Omega^{0} \times \mathbb{R}^{\ell}\right)} \text { for a.e. }(t, x, v) \in(0, T) \times \Omega^{t} \times \mathbb{R}^{\ell} .
$$

Indeed, consider the sequence $h_{n}:=f_{n+1}-f_{n}$, for all $n \geq 1$. By linearity, all the elements $h_{n}$ of the sequence satisfy (3.5)-(3.6)-(3.7) with initial condition $h_{1}=f^{\mathrm{in}}(x, v)$ and $h_{n}=0$ when $n \geq 2$, for a.e. $(x, v) \in \Omega^{0} \times \mathbb{R}^{\ell}$. In particular, we deduce that

$$
h_{n} \geq 0 \text { for a.e. }(t, x, v) \in(0, T) \times \Omega^{t} \times \mathbb{R}^{\ell} .
$$

We have hence built a monotone non-decreasing sequence $\left\{f_{n}\right\}_{n \in \mathbb{N}}$ composed by non-negative and uniformly bounded functions a.e. in the domain of definition of the problem. By consequence, the sequence $\left\{f_{n}\right\}_{n \in \mathbb{N}}$ pointwise converges to a limit $f$, which is by construction a solution of the initial-boundary value problem (2.5)-(2.6)-(2.7).

On the other hand, the sequence $\left\{f_{n}\right\}_{n \in \mathbb{N}}$ is composed of uniformly bounded functions thanks to (3.8) and hence the convergence to $f$ is not only pointwise, but also in the weak* sense.

By construction, the obtained solution of (2.5)-(2.6)-(2.7) is non-negative.

Thanks to Corollary 3.2, we have hence proved that he initial-boundary value problem (2.5)-(2.6)-(2.7) has one and only one solution, which is non-negative provided that the initial condition $f^{\text {in }}$ is non-negative. Eventually, the function $f$ inherit the properties of uniqueness and regularity proved in Lemma 3.1 and Corollary 3.2. This concludes the proof. 


\section{THE NUMERICAL METHOD}

In this section we describe the numerical strategy used for the study of (2.5)-(2.6)-(2.7), which is an extension of the particle method proposed in [8] by taking into account the effects of the dust particles on the gaseous particles.

In practice, a particle method simulates a population, composed of a great number of individual entities, by a reduced set of moving particles (whose number can be handled by a computing machine), which obey to the basic physical principles of the problem. For this reason, it allows the control of the main quantities used in the simulations, such as the mean free path between collisions, the particle free flow and the collision frequency.

In a particle method, the initial density $f^{\text {in }}$ of the gas is discretized by means of a collection of weighted smooth shape functions centered on the particle positions, that is

$$
f_{\varepsilon, N_{m}}^{\text {in }}(x, v)=\sum_{k=1}^{N_{m}} \omega_{k} \varphi_{\varepsilon}\left(x-x_{k}^{0}\right) \varphi_{\varepsilon}\left(v-v_{k}^{0}\right),
$$

where $N_{m}$ represents the number of numerical particles, $\omega_{k}$ is the weight of the $k$-th particle and the shape function $\varphi_{\varepsilon}(x)=\varphi\left(\varepsilon^{-1} x\right) / \varepsilon^{d}$ is a smooth function with compact support.

Roughly speaking, the weights $\omega_{k}$ mean that a numerical particle represents $\omega_{k}$ real molecules of the gas. The term "numerical particles" is here used for avoiding any confusion with the (real) number of dust particles.

Once the number $N_{m}$ of numerical particles has been chosen, the initial positions $\left(x_{k}^{0}\right)_{1 \leq k \leq N_{m}}$ and velocities $\left(v_{k}^{0}\right)_{1 \leq k \leq N_{m}}$ are sampled according to the initial density $f^{\text {in }}$. Then, the positions and velocities of the numerical particles evolve in time by taking into account the different phenomena listed below:

i) the free flow of the particles in the absence of any interaction, mathematically represented by the transport operator $v \cdot \nabla$;

ii) the specular reflexion of the gas particles at the boundary $\partial D$;

iii) the diffuse reflexion between gas particles and spherical dust particles;

$i v)$ the time evolution of the set of dust particles.

We introduce a time discretization of step $\Delta t$ and we set $t^{n}=n \Delta t$. The density of gaseous molecules at time $t^{n}$ - i.e. $f\left(t^{n}, \cdot, \cdot\right)$ where $f$ is the solution of $(2.5)-(2.6)-(2.7)$ - is then approached by

$$
f_{\varepsilon, N_{m}}^{n}(x, v)=\sum_{k=1}^{N_{m}} \omega_{k} \varphi_{\varepsilon}\left(x-x_{k}^{n}\right) \varphi_{\varepsilon}\left(v-v_{k}^{n}\right),
$$

where $\left(x_{k}^{n}\right)_{1 \leq k \leq N}$ and $\left(v_{k}^{n}\right)_{1 \leq k \leq N}$ are the positions and the velocities of the numerical particles at time $t^{n}$.

Let $N_{p}$ be the number of dust particles. The numerical strategy for describing the evolution of the mixture is the following.

At each time step $t^{n}$, for each numerical particle of position $x_{k}^{n}$ and velocity $v_{k}^{n}$, we set

$$
x_{k}^{n, 0}(t)=x_{k}^{n}+\left(t-t^{n}\right) v_{k}^{n}, \quad \text { for } t \in\left[t^{n}, t^{n}+\Delta t\right] .
$$

- If

$$
\min _{1 \leq i \leq N_{p}} \min _{t \in\left[t^{n}, t^{n}+\Delta t\right]}\left\|\xi_{i}(t)-x_{k}^{n, 0}(t)\right\| \leq r
$$

then we compute a collision between the macro particle labeled by the index $k$ and the dust particle labeled by the index $i_{0}$ such that

$$
i_{0}=\operatorname{argmin}_{1 \leq i \leq N_{p}} \min _{s \in\left[t^{n}, t^{n}+\Delta t\right]}\left\|\xi_{i}(t)-x_{k}^{n, 0}(t)\right\| .
$$

At time $t^{n, 1} \in\left[t^{n}, t^{n}+\Delta t\right]$ such that

$$
t^{n, 1}=\min \left\{t \in\left[t^{n}, t^{n}+\Delta t\right]:\left\|\xi_{i}(t)-x_{k}^{n, 0}(t)\right\|=r\right\}
$$


the velocity $v_{k}^{n}$ of macro-particle $k$ is modified in $v_{k}^{n, 1}$ thanks to a diffuse reflexion mechanism (see Appendix 1). A new trajectory is then introduced:

$$
x_{k}^{n, 1}(t)=x_{k}^{n, 0}\left(t^{n, 1}\right)+\left(t-t^{n, 1}\right) v_{k}^{n, 1}, \quad \text { for } t \in\left[t^{n, 1}, t^{n}+\Delta t\right] .
$$

- We compute the possible intersection between the trajectory of the macro-particle $\left(x_{k}^{n, 0}(t)\right.$ or $\left.x_{k}^{n, 1}(t)\right)$ and the boundary of the container $\partial D$. If the particle intersects the boundary $\partial D$ at time $t^{n, 2} \leq t^{n}+\Delta t$, we compute the new velocity $v_{k}^{n, 2}$ and trajectory $x_{k}^{n, 2}(t)$ after the treatment of the boundary condition.

- We compute in this way a sequence of trajectories $x_{k}^{n, 0}, x_{k}^{n, 1}, \ldots, x_{k}^{n, I}$, and velocities $v_{k}^{n, 0}:=v_{k}^{n}, v_{k}^{n, 1}, \ldots, v_{k}^{n, I}$, until $x_{k}^{n, I}(s)$ does not intersect $\partial \Omega^{t}$, for $t \in\left[t^{n, I}, t^{n}+\Delta t\right]$. We finally set $x^{n+1}=x_{k}^{n, I}, v^{n+1}=v_{k}^{n, I}, t^{n+1}=t^{n}+\Delta t$.

Note that this strategy can be easily parallelized, because of the lack of interactions between pairs of numerical gas particles. Only the reduction operations for computing the global quantities (such as the temperature) require to consider the global level.

\section{Numerical RESUlts}

We describe here a series of numerical experiments in dimension $\ell=2$, corresponding to some meaningful situations.

We suppose that the initial density is uniform in space and that it is described by a Maxwellian function in velocity, that is

$$
f^{\mathrm{in}}(x, v)=f^{\mathrm{in}}(v)=\frac{n_{0} m}{2 \pi k_{b} T^{\text {in }}} e^{-\frac{m\left|v-u^{\mathrm{in}}\right|^{2}}{2 k_{b} T^{\text {in }}}},
$$

where $m$ is the mass of a gas molecule, $u^{\text {in }}$ and $T^{\text {in }}$ are respectively the initial macroscopic velocity and the temperature of the gas (in $\mathrm{K}$ ), and $n_{0}$ correspond to $\left\|f^{\mathrm{in}}\right\|_{L^{1}\left(\Omega^{0} \times R^{3}\right)} /\left|\Omega^{0}\right|$. In this case, each component $\left(v_{k}^{0}\right)_{i}$, for $1 \leq k \leq N_{p}$ of the initial velocities of the gas particles is sampled according a gaussian law of mean $\left(u^{\text {in }}\right)_{i}$ and variance $k_{b} T^{\text {in }} / m$ (see [6] for details). The weights of the particles are identical, and are tuned in order to reproduce the mass of the initial condition:

$$
\omega_{k}=\left\|f^{\text {in }}\right\|_{L^{1}\left(\Omega^{0} \times R^{3}\right)} / N_{p}, \quad \text { for all } 1 \leq k \leq N_{p} .
$$

The initial positions of the numerical particles have been fixed on a regular grid. We clearly checked that no numerical particles were located insider a dust particle. Unless otherwise stated, the values of the physical quantities relative to the gas are taken as summarized in Table 5; here the mean free path $\lambda$ of the gas has the same order of magnitude of the width of the domain, so that the Knudsen number $K_{n}$ of the gas is equal to 2.5. Once the Mach number $M_{a}$ and the Temperature $T^{\text {in }}$ have been chosen, the macroscopic velocity of the gas is set equal to $u^{\text {in }}=a M_{a}$, where $a$ is the sound velocity in air at temperature $T^{\text {in }}$. The radius of the particles is $r=10^{-5} \mathrm{~m}$, and the other physical quantities depend on the scenarios considered in the next subsections. The domain $D$ is the square $[-1,1] \times[-1,1]\left(\right.$ in $\left.10^{-4} \mathrm{~m}\right)$,

$$
\begin{array}{cccccc}
\lambda & K_{n} & n_{0} & T^{\text {in }} & M_{a} & u^{\text {in }} \\
5 \times 10^{-4} \mathrm{~m} & 2.5 & 2 \times 10^{22} & 293 \mathrm{~K} & 0.1 & 34.41 \mathrm{~m} / \mathrm{s}
\end{array}
$$

TABLE 1. Physical quantities relative to the gas.

with specular reflexion at the boundary. However, in Scenario 1 (Figure 3), we use a periodic boundary condition at the left and at the right sides of $\partial D$, in order to mimic an infinite domain in the $x$ direction.

We use $B_{3}$-splines (see [6]) as shape functions $\varphi$, with a shape size $\varepsilon=h^{0.4}$, where $h$ is the initial distance between two numerical gas particles in each direction (and which is obviously linked to $N_{m}$ ). In Scenario 1 we use $N_{m}=62994696$ numerical particles, and we use $N_{m}=246092$ in the other scenarios. The time step is $\Delta t=10^{-9} \mathrm{~s}$. 
Scenario 1. The first simulation describes the evolution of a system of two particles with translational velocities $u_{1}=\left(0, u_{d}\right)$ and $u_{2}=\left(0,-1.5 u_{d}\right)$, with $u_{d}=2 u^{\text {in }}$, and no rotational velocities. The temperature of the surface of particles is $T_{p}=500 \mathrm{~K}$. The macroscopic velocity of the gas is $u_{g}=\left(-u^{\text {in }}, 0\right)$. In Figure 2, we plot the time evolution of the number density

$$
\rho(t, x)=\int_{\mathbb{R}^{3}} f(t, x, v) d v
$$

of the gas at various time instants and, in Figure 3, the evolution of the gas temperature

$$
T(t, x)=\frac{m}{2 k_{B} \rho(t, x)}\left[\int_{\mathbb{R}^{3}} f(t, x, v) v^{2} d v-\left|\frac{1}{\rho(t, x)} \int_{\mathbb{R}^{3}} f(t, x, v) v d v\right|^{2}\right] .
$$

The physical values are scaled according to the length scale $L^{\circ}=10^{-4} \mathrm{~m}$ and the number density scale $d^{\circ}=2 \times 10^{22}$ molecules.

Scenario 2. In Figure 4 (left), we plot the time evolution of the mean temperature of the gas

$$
\langle T(t)\rangle=\int_{\Omega^{t}} T(t, x) d x
$$

with a motionless particle at temperature $T_{p}=500 \mathrm{~K}$.

Scenario 3. In Figure 4 (right), we plot the time evolution of the mean temperature of the gas with a motionless particle at temperature $T_{p}=100 \mathrm{~K}$. We can compare the behavior of the mean temperature in Scenarios 2 and 3. In both situations, the temperature of the rarefied carrying gas quickly approaches the (constant) temperature of the dust particle.

Scenario 4. The setting of Scenario 4 is very similar to Scenario 3. The only difference with respect to the previous scenario is that the spherical dust particle has a rotational velocity equal to $2 \pi \times 10^{6} \mathrm{rad} \cdot \mathrm{s}^{-1}$. The time evolution of the mean temperature of the gas is compared with the situation of Scenario 3 in Figure 5 (left). We can recognize the effect of the rotational velocity of the dust particle to the temperature of the rarefied gas mixture.

Scenario 5. In Scenario 5, we consider $T_{p}=T^{\text {in }}=293 \mathrm{~K}$ and $u^{\text {in }}=0$. In this case (Figure 5, right), the time evolution of the temperature of the gas is the pure effect of the energy transfer via the rotational velocity (equal to $2 \pi \cdot 10^{6} \mathrm{rad} \cdot \mathrm{s}^{-1}$ ). The number of numerical molecules is here 992140.

\section{APPENDIX 1: NUMERICAL TREATMENT OF THE DIFFUSE REFLEXION MECHANISM}

We describe here the treatment of the boundary condition (2.7) on the moving boundary $\Gamma^{t}$, adapted from the treatment of a diffuse reflexion condition on a fixed boundary at temperature $T_{p}$ (see [12]). For the sake of simplicity, we take $m=1$ and $k_{B}=1$ in the following. If a numerical particle hits the boundary $\Gamma^{t}$ at $x$, then its velocity will be generated by sampling from the half Maxwellian $h(v-c(t, x))$, where

$$
h(v)=\frac{(2 \pi)^{\frac{3-\ell}{2}}}{T_{p}^{\frac{\ell-1}{2}}} M_{T_{p}}(v)\left|v \cdot n_{x}\right| \mathbb{1}_{\left\{v \cdot n_{x} \geq 0\right\}},
$$

where $\ell$ is the dimension $(\ell \in\{2,3\})$. Because of its normalization, $h$ is a probability density. If we note $v=\left(v_{1}, v_{\perp}\right)$, with $v_{1}=v \cdot n_{x}$, then $h$ can be written as the product $h(v)=$ $h_{1}\left(v_{1}\right) h_{2}\left(v_{\perp}\right)$, with

$$
h_{1}(s)=\frac{1}{T_{p}} s \exp \left(-\frac{s^{2}}{2 T_{p}}\right) \mathbb{1}_{\{s \geq 0\}}, s \in \mathbb{R}, \text { and } h_{2}(w)=\frac{1}{\left(2 \pi T_{p}\right)^{l / 2}} \exp \left(-\frac{|w|^{2}}{2 T_{p}}\right), w \in \mathbb{R}^{\ell-1} .
$$

The repartition function $\psi_{1}(s)=1-\exp \left(-s^{2} / 2 T_{p}\right)$ of $h_{1}$ is invertible, and

$$
\psi_{1}^{-1}(u)=\sqrt{-2 T_{p} \ln (1-u)} \text {. }
$$



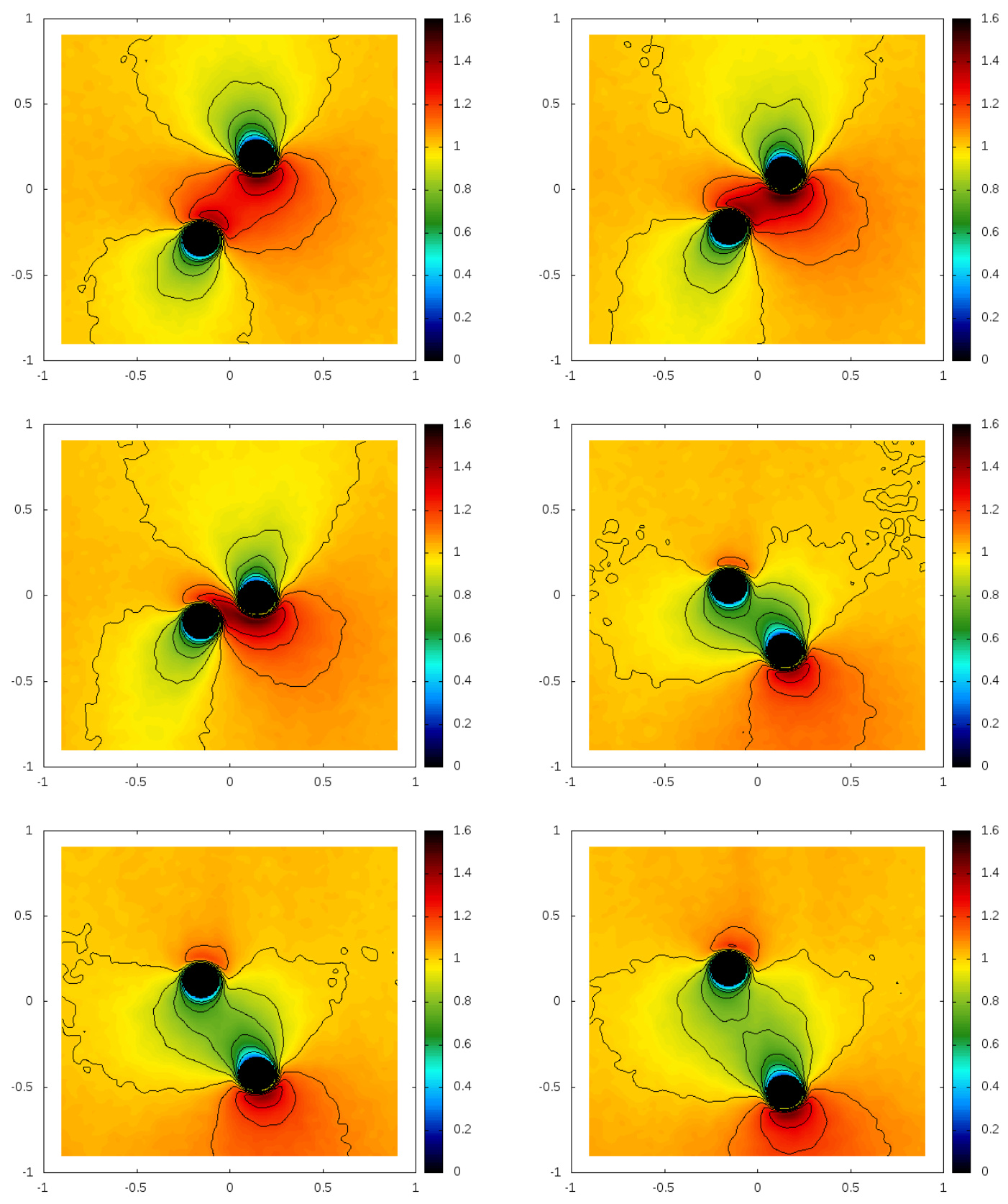

Figure 2. Time history (from the left to the right and from the top to the bottom) of the gas density in Scenario 1 at times $t_{3}=3.0 \times 10^{-7} \mathrm{~s}, t_{4}=$ $4.0 \times 10^{-7} \mathrm{~s}, t_{5}=5.0 \times 10^{-7} \mathrm{~s}, t_{8}=8.0 \times 10^{-7} \mathrm{~s}, t_{9}=9 \times 10^{-7} \mathrm{~s}, t_{10}=1.0 \times 10^{-6}$ $\mathrm{s}$.

Assume now $l=2$. As we cannot explicitly express the repartition function $\psi_{2}$ of $h_{2}$, we use the classical trick to simulate a Gaussian random variable, by working with polar variables $\left(w_{1}, w_{2}\right) \rightarrow(R, \theta)$, with $w_{1}=R \cos (\theta)$ and $w_{2}=R \sin (\theta)$ - in the joint density of $\left(w_{1}, w_{2}\right)$ :

$$
\psi_{2}\left(w_{1}\right) \psi\left(w_{2}\right) d w_{1} d w_{2}=\frac{1}{T_{p}} R \exp \left(-R^{2}\right) d R \frac{d \theta}{2 \pi} .
$$

This allow to invert the repartition function of the variable $R$. We then randomly compute $V_{r}=\left(V_{r 1}, V_{r \perp}\right)$ following the law $h$ by sampling three independent random variables $U_{1}, U_{2}$ 

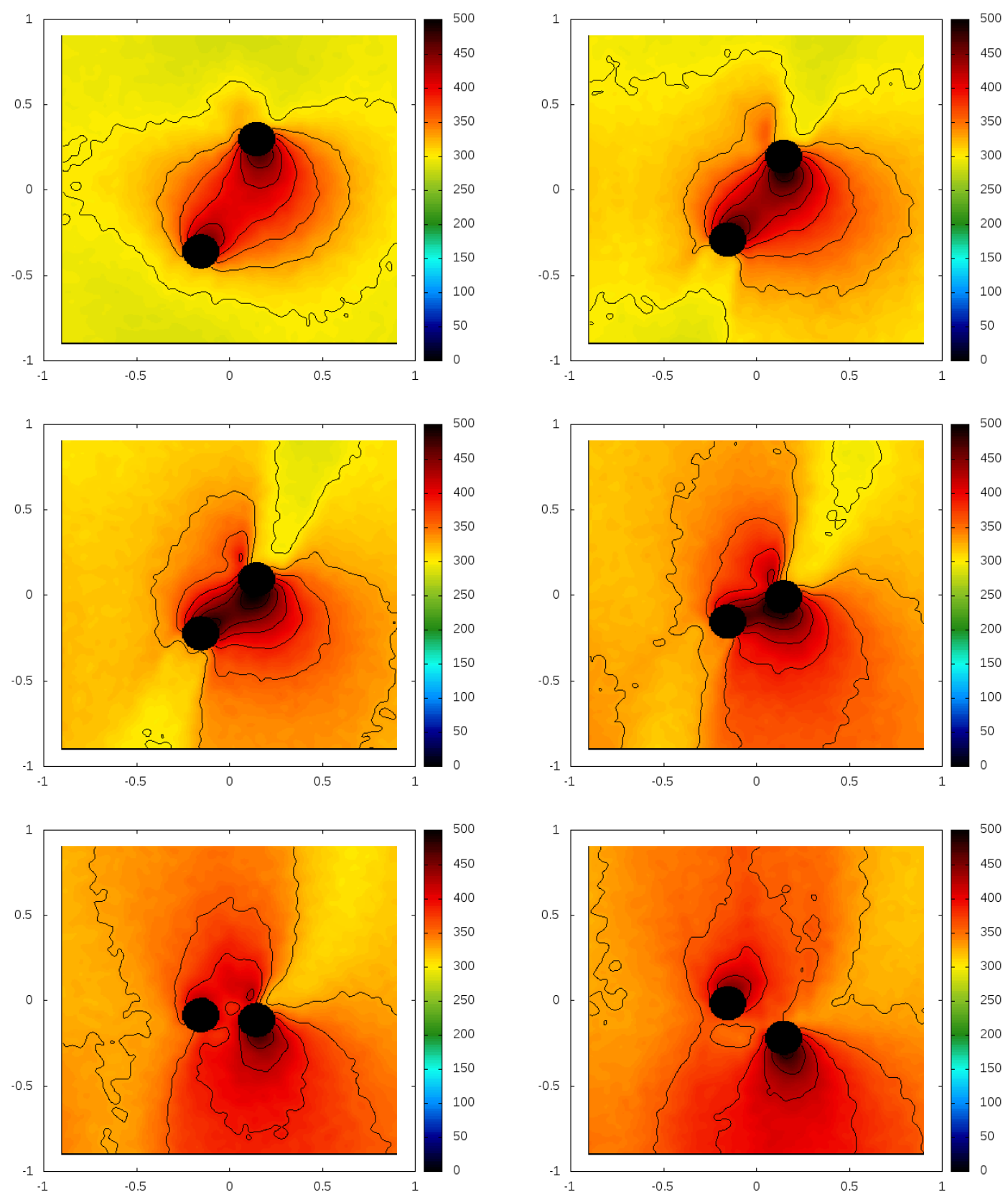

Figure 3. Time history (from the left to the right and from the top to the bottom) of the gas temperature in Scenario 1 at times $t_{2}=2.0 \times 10^{-7} \mathrm{~s}$, $t_{3}=3.0 \times 10^{-7} \mathrm{~s}, t_{4}=4.0 \times 10^{-7} \mathrm{~s}, t_{5}=5.0 \times 10^{-7} \mathrm{~s}, t_{6}=6 \times 10^{-7} \mathrm{~s}$, $t_{7}=7.0 \times 10^{-7} \mathrm{~s}, t_{8}=8.0 \times 10^{-7} \mathrm{~s}$.

and $U_{3}$, following a uniform law on $[0,1]$, and set

$$
V_{r 1}=\sqrt{-2 T_{p} \ln \left(1-U_{1}\right)}, \quad \text { and } \quad V_{r \perp}=\sqrt{-2 T_{p} \ln \left(1-U_{2}\right)} \cos \left(2 \pi U_{3}\right) \text {. }
$$

Finally the velocity of the numerical particle after reflexion on boundary $\Gamma^{t}$ is set to

$$
v_{a r}=c(t, x)+\left(V_{r 1}, V_{r \perp}\right) .
$$



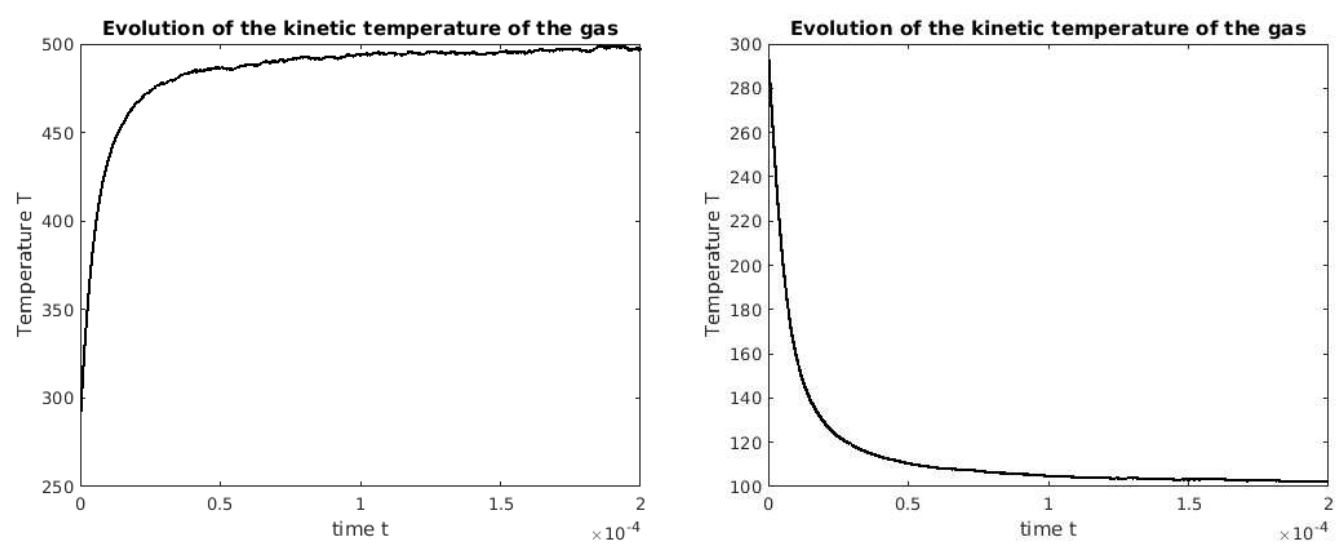

Figure 4. Time history of the mean temperature in Scenario 2 (left) and Scenario 3 (right).
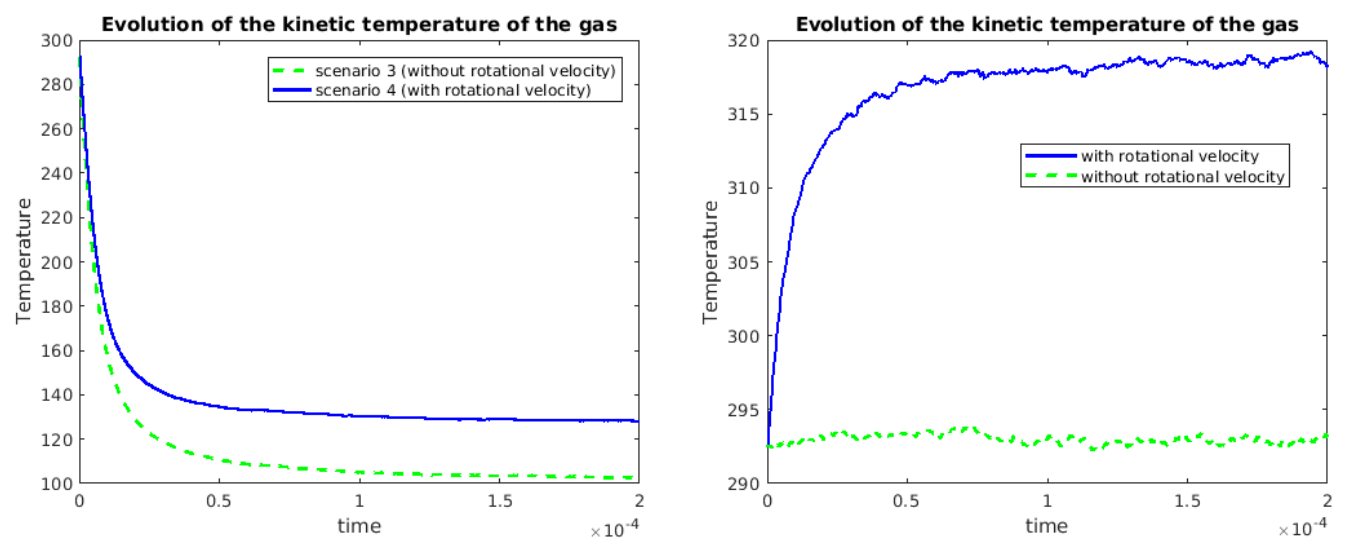

Figure 5. Comparison of the evolution of the mean temperature in Scenario 3 and Scenario 4 (left), and evolution of temperature in Scenario 5, with and without rotational velocity (right).

Acknowledgements. This work has been carried out in the framework of the projects Kimega (ANR-14-ACHN-0030-01), PHC Sakura "Dynamics of small particles in fluids: modeling and numerics" and of the Galileo program of the Università Italo-Francese. This research was moreover supported by the Italian Ministry of Education, University and Research (MIUR), Dipartimenti di Eccellenza Program - Dept. of Mathematics "F. Casorati", University of Pavia.

\section{REFERENCES}

[1] Kazuo Aoki and François Golse. On the speed of approach to equilibrium for a collisionless gas. Kinet. Relat. Models, 4(1):87-107, 2011.

[2] Étienne Bernard and Francesco Salvarani. On the convergence to equilibrium for degenerate transport problems. Arch. Ration. Mech. Anal., 208(3):977-984, 2013.

[3] Étienne Bernard and Francesco Salvarani. On the exponential decay to equilibrium of the degenerate linear Boltzmann equation. J. Funct. Anal., 265(9):1934-1954, 2013.

[4] Étienne Bernard and Francesco Salvarani. Optimal estimate of the spectral gap for the degenerate Goldstein-Taylor model. J. Stat. Phys., 153(2):363-375, 2013.

[5] Stefanella Boatto and François Golse. Diffusion approximation of a Knudsen gas model: dependence of the diffusion constant upon the boundary condition. Asymptot. Anal., 31(2):93-111, 2002.

[6] Frédérique Charles, Cédrick Copol, Stéphane Dellacherie, and Jean-Marc Mounsamy. Numerical simulation by a random particle method of deuterium-tritium reactions in a plasma. ESAIM PROCEEDINGS, 38:220$240,2012$. 
[7] J Darrozes and JP Guiraud. Généralisation formelle du théorème h en présence de parois. applications. Comptes rendus hebdomadaires des séances de l'Académie des sciences série A, 262(24):1368, 1966.

[8] Florian De Vuyst and Francesco Salvarani. Gpu-accelerated numerical simulations of the knudsen gas on time-dependent domains. Comput. Phys. Comm., 184(3):532-536, 2013.

[9] Florian De Vuyst and Francesco Salvarani. Numerical simulations of degenerate transport problems. Kinet. Relat. Models, 7(3):463-476, 2014.

[10] Daniel Han-Kwan and Matthieu Léautaud. Geometric analysis of the linear Boltzmann equation I. Trend to equilibrium. Ann. PDE, 1(1):Art. 3, 84, 2015.

[11] Francesco Salvarani. On the linear Boltzmann equation in evolutionary domains with an absorbing boundary. J. Phys. A, 46(35):355501, 15, 2013.

[12] Yoshio Sone. Molecular gas dynamics. Modeling and Simulation in Science, Engineering and Technology. Birkhäuser Boston, Inc., Boston, MA, 2007. Theory, techniques, and applications.

[13] Tetsuro Tsuji, Kazuo Aoki, and François Golse. Relaxation of a free-molecular gas to equilibrium caused by interaction with vessel wall. J. Stat. Phys., 140(3):518-543, 2010.

Laboratoire Jacques-Louis Lions, UPMC \& CNRS UMR 7598, Sorbonne Université, F-75005 PARIS, FRANCE

Email address: charles@ljll.math.upmc.fr

F.S.: Université Paris-Dauphine, Ceremade, UMr CNRS 7534, F-75775 Paris Cedex 16, France

\& Università degli Studi di Pavia, Dipartimento di Matematica, I-27100 Pavia, Italy

Email address: francesco.salvarani@unipv.it 